Document downloaded from:

http://hdl.handle.net/10251/154102

This paper must be cited as:

Fernandez-Arroyo, A.; Delgado-Muñoz, D.; Domine, ME.; López Nieto, JM. (2017). Upgrading of oxygenated compounds present in aqueous biomass-derived feedstocks over NbOx-based catalysts. Catalysis Science \& Technology. 7(23):5495-5499. https://doi.org/10.1039/c7cy00916j

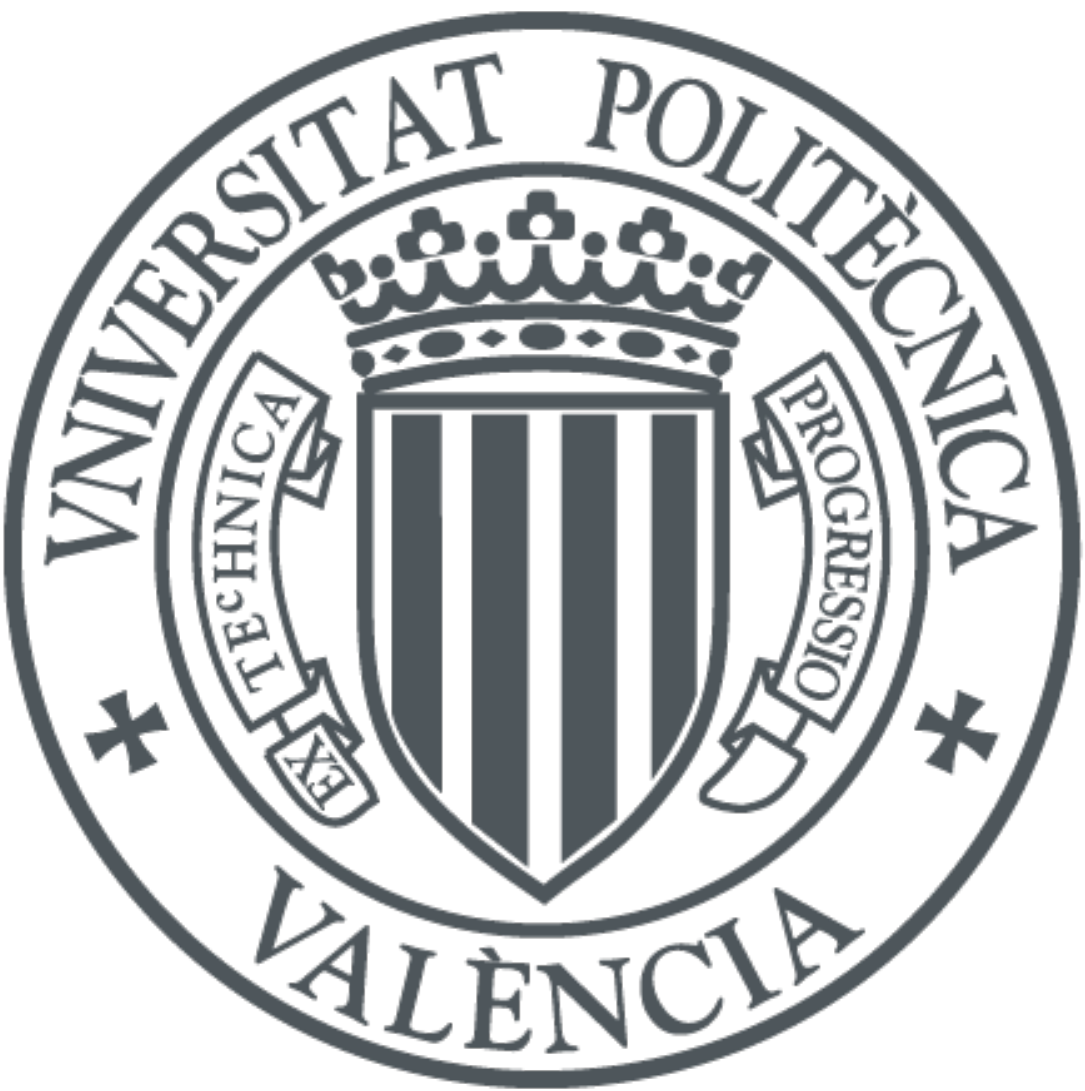

The final publication is available at

https://doi.org/10.1039/c7cy00916j

Copyright The Royal Society of Chemistry

Additional Information 


\title{
Upgrading of oxygenated compounds present in aqueous biomass-derived feedstocks over NbOx-based catalysts
}

\author{
A. Fernández-Arroyo, ${ }^{a}$ D. Delgado, ${ }^{a}$ M. E. Domine, ${ }^{* a}$ and J. M. López-Nieto*a
}

Instituto de Tecnología Química (UPV-CSIC)

Universitat Politècnica de València- Consejo Superior de Investigaciones Científicas.

Avenida de los Naranjos s/n, 46022 Valencia (Spain)

E-mail:mdomine@itq.upv.es; jmlopez@itq.upv.es.

\begin{abstract}
THE INFLUENCE OF SYNTHESIS AND POST-SYNTHESIS PROCEDURES OF DIFFERENT NIOBIUM OXIDES ON THEIR CATALYTIC PERFORMANCE IN THE AQUEOUS PHASE CONDENSATION OF OXYGENATED COMPOUNDS IS STUDIED. HYDROTHERMALLY SYNTHESIZED NIOBIUM OXIDE WITH A PSEUDO-CRYSTALLINE STRUCTURE SHOWS ENHANCED ACID PROPERTIES, SURFACE AREA AND CONSEQUENTLY BETTER CATALYTIC ACTIVITY THAN NB2 $\mathrm{O}_{5}$ PREPARED BY OTHER SYNTHESIS METHODS. THE OPTIMIZED NBOX-BASED CATALYST ALSO DEMONSTRATES HIGHER STABILITY AFTER SEVERAL REUSES COMPARED TO CE-ZR MIXED OXIDE REFERENCE CATALYST.
\end{abstract}




\section{Manuscript}

In the last years lignocellulosic biomass valorisation has become a sustainable alternative to the use of fossil sources for the production of fuels and chemicals. Thus, the use of this type of biomass as raw material represents a potential solution to environmental issues derived from the use of oil and its depletion. ${ }^{1-4}$ In this context, and after a primary treatment of biomass via fast pyrolysis processes, bio-oils are predominantly obtained. ${ }^{3,4}$ These bio-oils are complex mixtures containing water and oxygenated organic compounds in varying concentrations, which are characterized by their high reactivity. This hinders their storage and direct use as liquid fuel. Therefore, an upgrading approach is needed to improve their fuel properties (O content reduction, water elimination, etc.). Most common upgrading process includes bio-oil hydrotreating with NiMoS/ and CoMoS $/ \mathrm{Al}_{2} \mathrm{O}_{3}$ industrial catalysts at high $\mathrm{H}_{2}$ pressures (>60-70 bar) and temperatures (350-450 으)..$^{5,6}$ Resulting oil possesses better fuel quality but hydrogen and energy consumptions during the process are quite high, while liquid productivities are low mainly due to $\mathrm{C} 1-\mathrm{C} 4$ highly abundant oxygenated compounds are converted into gases during the process. Thermal and catalytic cracking of bio-oils have been also intended, ${ }^{7-9}$ as well as co-processing of bio-oils with petroleum fractions mainly in FCC units. Results obtained show that at least a partial upgrading of bio-oil is needed before co-processing. ${ }^{10}$ Alternatively, a liquid-liquid separation process by water addition to the pyrolytic bio-oils is also feasible ${ }^{11}$. Then separated organic fractions are further processed for their application as liquid fuels, whereas aqueous fractions containing $\mathrm{C} 1-\mathrm{C} 4$ acids (i.e. acetic acid), aldehydes, ketones, alcohols and low amounts of heavier watersoluble compounds, constitute nowadays waste effluents at bio-refineries. ${ }^{11,12}$ Aqueous phase reforming (APR) approach developed by Dumesic and coworkers ${ }^{13,14}$ could be applied to produce $\mathrm{H}_{2}$ from these aqueous fractions, although with high energy consumption, and more importantly, by losing all Carbon derived from biomass (low atom economy).

Aligned with the new bio-economy concept ${ }^{15}$, the transformation of these lowvalue water-soluble compounds would be of great interest. Indeed, they can be 
transformed into a mixture of hydrocarbons and aromatics useful for blending with automotive fuels. In a first step, this can be achieved performing "one pot" $\mathrm{C}-\mathrm{C}$ bonds formation reactions, such as aldol condensation and ketonization, among others. ${ }^{16}$ The importance of ketonization lies in its capacity for reducing carboxylic acids content whilst condensation reactions allow consecutively $\mathrm{C}-\mathrm{C}$ bonds formation. Both reaction mechanisms have been widely studied by using probe molecules as reactants ${ }^{17,18}$ concluding that Lewis acid/base sites combination is necessary to assist intermediates formation on the surface of the catalyst. In this context, catalysts based on Ce-Zr-O mixed oxides have been extensively considered ${ }^{18}$ en-for the gas-phase conversion of small aldehydes in the presence of acids and water at high temperatures $(>300 \circ C)$. More recently, $\mathrm{TiO}_{2}$ or $\mathrm{ZrO}_{2}$ have been studied as catalysts for condensation reactions using acetic acid or propanal as probe molecules, ${ }^{19,20}$ and even with the incorporation of noble metals on the solid catalyst. ${ }^{21}$ In general, the activity of these catalysts is based on their bifunctional character, although their stability under faithful operation conditions is a critical factor to be further improved.

Based on these considerations, the stability and acid properties of niobium oxides at high reaction temperatures (even in the presence of water) make these materials attractive for industrial use as heterogeneous catalysts: ${ }^{22}$ i) they show great stability against acids; ii) most of their Lewis acid sites remain active even in aqueous environments when tested in different reactions (i.e. acetalization, isomerization). ${ }^{23,24}$ Moreover, crystalline structure, surface area and acid properties can be modified depending on the synthesis procedure and/or the post-synthesis thermal treatment. ${ }^{25,26}$ Specifically, we have found that both the Brönsted/Lewis ratio and the total number of acid sites in niobium-containing materials can be tuned by an appropriate combination of hydrothermal synthesis and post-synthesis treatments, thus improving both the acid characteristics and the catalytic performance of $\mathrm{NbOx}$-based catalysts. ${ }^{27}$

In this work, we show the influence of both the synthesis procedure and the heattreatment conditions on the catalytic performance of different niobium oxides in the valorisation of aqueous effluents obtained by liquid phase separation of 
pyrolytic bio-oils. The catalytic behaviour of $\mathrm{NbOx}$-catalysts is investigated by using an aqueous mixture of C2-C3 representative oxygenated compounds, closer to the real conditions at industry and differing from usual probe molecules studies performed even in the absence of water in most of the cases. The catalytic results are compared with a Ce-Zr-O mixed oxide, which is the reference material in literature. ${ }^{18}$

$\mathrm{Nb}$-oxide based catalysts were prepared by hydrothermal synthesis ${ }^{27}$ (HT-series ${ }^{\S}$ ). For comparative purposes, niobium oxides prepared by conventional precipitation (PR-series) and commercial $\mathrm{Nb}_{2} \mathrm{O}_{5}$ from Sigma-Aldrich (C-series), have been also tested (See SI). All the niobium-based samples were heat-treated in air or $\mathrm{N}_{2}$ to obtain the final catalysts. The catalysts are named as HT-xy, PR-xy and C-xy (for hydrothermal, precipitation or commercial materials, respectively), where $\mathrm{x}$ is temperature and $\mathrm{y}$ is $\mathrm{A}$ or $\mathrm{N}$, depending on the atmosphere (air or $\mathrm{N}_{2}$, respectively) used during the heat-treatment. In addition, a $\mathrm{Ce}_{0.5} \mathrm{Zr}_{0.5} \mathrm{O}_{2}$ mixed oxide (CeZrO) was prepared via co-precipitation following the recipe of ref. ${ }^{28}$ (See $\mathrm{SI})$.

The catalysts were characterized by XRD, TEM, TG, EA, FT-IR of adsorbed pyridine, and $\mathrm{N}_{2}$-adsorption. Important differences in the crystallinity and particle size of the materials have been observed depending on the preparation procedure and the heat-treatment (Fig.1, see also Fig.S3 in SI for the rest of materials). In this sense, HT-series and PR-series materials heat-treated in $\mathrm{N}_{2}$, tend to form a pseudo-crystalline (Fig. 1a) or an amorphous phase (Fig.1b) respectively, while Cseries samples give rise to a well-ordered $\mathrm{T}-\mathrm{Nb}_{2} \mathrm{O}_{5}$ phase (JCPDS: 00-027-1313) (Fig.1C). In addition, oxides prepared by hydrothermal method show the lowest particle size (ca.10-20 nm) (Fig.1a).

Pseudo-crystalline materials obtained through hydrothermal procedure present Bragg signals corresponding to 001 and 002 planes in a crystal structure ordered along c-axis (c = $3.9 \AA$ ), which is related to $\mathrm{ReO}_{3}$-type structure (Fig. S3 and S5, SI), as it has been observed in other NbOx-based materials. ${ }^{29,30}$ It is noteworthy to mention that heat-treatments in air promote the crystallization, at least partially of the orthorhombic $\mathrm{T}_{-} \mathrm{Nb}_{2} \mathrm{O}_{5}$ phase (JCPDS: 00-027-1313) (Fig. S3, SI). On the 
other hand, $\mathrm{C}-\mathrm{Nb}_{2} \mathrm{O}_{5}$ samples show a well-ordered crystal structure regardless the heat-treatment atmosphere (Fig. S3 and S5, SI).

Catalytic results of selected catalysts tested in the transformation of oxygenated compounds present in an aqueous model mixture including acetic acid, ethanol, propanal and acetol (liquid phase system in an autoclave reactor at $200{ }^{\circ} \mathrm{C}$ and $\mathrm{P}_{\mathrm{N} 2}$ $=13$ bar during 7h) are shown in Table 1. Results expressed in terms of total organic yield and yield to the main reaction products are calculated by considering that the maximum attainable value of total organic products for the composition of the aqueous mixture studied is $\approx 30 \%$ (See $\mathrm{SI}$ ). As a reference, catalytic performance of CeZrO has also been included.

Additionally, the effect of the atmosphere (air or $\mathrm{N}_{2}$ ) used during heat-treatment on the catalytic activity has been also studied (Table S2, SI). In all cases, higher organic products yields are observed when the heat-treatment is carried out in $\mathrm{N}_{2}$ atmosphere (Table S2, SI).

More importantly, hydrothermally synthesized niobium oxides (HT-series) reached higher propanal conversion (>90\%) and 2-metil-2-pentenal (2M2P) yield (Table S2, SI), thus achieving the highest total organic products yields (Table 1).

A deeper understanding about HT-series catalytic activity can be achieved by individually studying the behaviour of reactants conversion present in the aqueous model mixture (Fig. 2a). Complete conversion of acetol and high conversion of Based on these considerations, the stability and acid properties of niobium oxides at high reaction temperatures (even in the presence of water) make these materials attractive for industrial use as heterogeneous catalysts: ${ }^{22}$ i) they show great stability against acids; ii) most of their Lewis acid sites remain active even in aqueous environments when tested in different reactions (i.e. acetalization, isomerization). ${ }^{23,24}$ Moreover, crystalline structure, surface area and acid properties can be modified depending on the synthesis procedure and/or the post-synthesis thermal treatment. ${ }^{25,26}$ Specifically, we have found that both the Brönsted/Lewis ratio and the total number of acid sites in niobiumcontaining materials can be tuned by an appropriate combination of 
hydrothermal synthesis and post-synthesis treatments, thus improving both the acid characteristics and the catalytic performance of NbOx-based catalysts. ${ }^{27}$

In this work, we show the influence of both the synthesis procedure and the heattreatment conditions on the catalytic performance of different niobium oxides in the valorisation of aqueous effluents obtained by liquid phase separation of pyrolytic bio-oils. The catalytic behaviour of $\mathrm{NbOx}$-catalysts is investigated by using an aqueous mixture of $\mathrm{C2}-\mathrm{C} 3$ representative oxygenated compounds in water, closer to the real conditions at industry and differing from usual probe molecules studies performed even in the absence of water in most of the cases. The catalytic results are compared with a Ce-Zr-O mixed oxide, which is the reference material in literature. ${ }^{18}$

Catalytic results of selected catalysts tested in the transformation of oxygenated compounds present in an aqueous model mixture including acetic acid, ethanol, propanal and acetol (liquid phase system in an autoclave reactor at $200 \circ \mathrm{C}$ and $\mathrm{P}_{\mathrm{N} 2}$ $=13$ bar during $7 \mathrm{~h}^{\S \S}$ ) are shown in Table 1. Results expressed in terms of total organic yield and yield to the main reaction products are calculated by considering that the maximum attainable value of total organic products for the composition of the aqueous mixture studied is $\approx 30 \%$ (See $\mathrm{SI}$ ). As a reference, catalytic performance of CeZrO has also been included.

Additionally, the effect of the atmosphere (air or $\mathrm{N}_{2}$ ) used during heat-treatment on the catalytic activity has been also studied (Table S2, SI). In all cases, higher organic products yields are observed when the heat-treatment is carried out in $\mathrm{N}_{2}$ atmosphere (Table S2, SI).

More importantly, hydrothermally synthesized niobium oxides (HT-series) reached higher propanal conversion (>90\%) and 2-metil-2-pentenal (2M2P) yield (Table S2, SI), thus achieving the highest total organic products yields (Table 1).

A deeper understanding about HT-series catalytic activity can be achieved by individually studying the behaviour of reactants conversion present in the aqueous model mixture (Fig. 2a). Complete conversion of acetol and high conversion of 
propanal are reached in short times, while ethanol and acetic acid exhibit lower conversion ( $\approx 50 \%$ and $15 \%$, respectively).

It is worth noting that the low acetic acid conversion was expected under the applied moderate reaction conditions, mainly due to the strong competition for the active sites of more reactive molecules, such as propanal and acetol present in the mixture. Thus, the study of catalyst performance by using complex mixtures is essential for the development of future industrial applications. In addition, the identification of main reaction products and their distribution profile observed by GC-MS (Fig. S2, SI) allow us to draw the evolution of products yield with reaction time when HT-450N was used (Fig. 2b). With all this information a reaction network is proposed (Fig. 3, see also SI), where ethyl acetate (from esterification reaction of acetic acid and ethanol), acetone (acetic acid ketonization product), main intermediate products (C5-C8) and final products (C9-C10) are highlighted.

As can be seen in Fig. $2 b$, high products yields are reached within 3 hours of reaction due to fast condensation reactions among propanal and acetol, whereas ethyl acetate is produced from acetic acid and ethanol esterification. Ethyl acetate yield maintains constant in time, meanwhile $2^{\text {nd }}$ condensation steps ocurr as intermediate C5-C8 products (mainly derived from acetol and propanal crosscondensation) keep reacting with propanal or other intermediate products (acetone, 3-pentanone, etc.) to generate larger (C9-C10) products (Fig. 2b, and Fig. S8).

The activity of HT-series $\mathrm{Nb}$-based catalysts in these ketonization and aldol condensation reactions is based on their improved acid and structural properties. Therefore, catalytic results can be explained taking into account the higher surface areas $\left(>70 \mathrm{~m}^{2} / \mathrm{g}\right)$ and the specific acid properties of the HT-series catalysts (Fig. S6, SI), which give rise to higher initial reaction rates (Table 1). In this way, the density of acid sites increases when decreasing the heat-treatment temperature, despite the increment of the surface area observed (Table 1). Indeed, the higher amount of total acid sites and their right distribution on the catalyst surface leads to increase the organic products yields during the process (Fig. 4). The optimal combination of these parameters has been found for HT- 
400N and HT-450-N samples, which leads to a better catalytic behaviour in terms of total organic yield than CeZrO reference catalyst (Table 1 and Fig. 4).

Interestingly, in spite of the higher density of acid sites and initial reaction rate observed for the sample heat-treated at the lowest temperature (i.e. sample HT$300 \mathrm{~N})$, its higher acidity could also favour undesired polymerization reactions during the process; thus lowering the final total organic products found in the liquid reaction mixture (Table 1 and Fig. S7, SI). This effect can be also observed by the decrease of the carbon balance when HT-300N was used (Table 1).

Finally, recycling experiments were performed testing selected catalysts during three consecutive reuses to corroborate the resistance of $\mathrm{Nb}$-oxides under reaction conditions ${ }^{\S \S}($ See $\mathrm{SI})$, and the results in terms of total organic yield attained are given in Fig. 5. On one hand, it was observed that HT-450N catalyst maintained its performance practically invariable after reuses due to structure preservation, this being confirmed by X-ray diffraction and Raman spectroscopy measurements (see $\mathrm{SI}$ ). On the other hand, C-450N sample exhibited high stability (low carbon deposition), although it catalytic activity decreased probably due to surface reconstruction/changes processes after being used in aqueous solutions (Figure S9, SI). In both $\mathrm{Nb}$-based catalysts, no leaching of $\mathrm{Nb}$ was observed. Remarkably, hydrothermally prepared catalysts still showed much better stability than CeZrO mixed oxide, which suffered a bigger loss of activity due to carbon deposition as it was measured by TG and EA (See SI), but mostly due to the significant leaching of $\mathrm{Ce}$ (>30wt.\% lost from the initial content in solid) clearly detected in liquids after reaction (See SI).

Although further studies must be performed, these results are very promising and open up new possibilities for the application of niobium oxide-based materials as catalysts for the condensation of oxygenated compounds present in complex aqueous mixtures.

\section{Conclusions}

Summarizing, niobium oxide based catalysts prepared by hydrothermal synthesis with a pseudo-crystalline structure (ordered just along c-axis) and showing 
tunable acid properties, have resulted to be active and selective catalysts for the valorization of oxygenated compounds in aqueous effluents obtained by phase separation of pyrolytic bio-oils. These optimized NbOx-materials present higher stability (after several reuses) and catalytic activity than those previously reported by other authors, mainly due to they are highly water resistant catalysts.

\section{Acknowledgements}

Financial support by Spannish Government (CTQ-2015-67592, CTQ-2015-68951C3-1, and SEV-2012-0267) is gratefully acknowledged. A.F-A. and D.D. thank "La Caixa-Severo Ochoa" Foundation and Severo Ochoa Excellence Program (SVP2014-068669), respectively, for their fellowships. Authors also want to thank the Electron Microscopy Service of Universitat Politècnica de València for their kind support.

\section{Notes and references}

$\S$ Catalysts Preparation: Hydrothermal Nb oxide (HT-series) ${ }^{27}$ was prepared with Nb oxalate monooxalate adduct (ABCR) (30.6 g) dissolved in distilled water (63,2 g) and kept $10 \mathrm{~min}$. under stirring at $80^{\circ} \mathrm{C}$. This solution was introduced in a Teflon-lined steel autoclave and kept at 175 으 for 2 days. The resulting solid was filtered and treated at 100 으 for $16 \mathrm{~h}$. Additional $\mathrm{Nb}$ oxide samples prepared by conventional precipitation (PR-series) and commercial $\mathrm{Nb}_{2} \mathrm{O}_{5}$ from Sigma-Aldrich (C-series) have been also tested after heat-treatment (See SI). All the Nb-based samples were heattreated in air or $\mathrm{N}_{2}$ at $300-550$ o $\mathrm{C}$ for $2 \mathrm{~h}$ prior to their use as catalysts. Ce-Zr mixed oxide ( $\left.\mathrm{Ce}_{0.5} \mathrm{Zr}_{0.5} \mathrm{O}_{2}, \mathrm{CeZrO}\right)$ was prepared via co-precipitation following the recipe of ref. ${ }^{28}$, and calcined in air at 450 으 during $1 \mathrm{~h}$ (See SI).

$\S \S$ Catalytic Experiments: Reactions were performed in an autoclave-type reactor by feeding $3.0 \mathrm{~g}$ of an aqueous model mixture composed by water (30 wt\%), acetic acid (30 wt\%), propanal (25 wt\%), acetol (5 wt\%), ethanol (10 wt\%), with $0.15 \mathrm{~g}$ of catalyst (See SI). The reactor was pressurized at 13 bar $\mathrm{N}_{2}$ and heated at 200 oC under continuous stirring. Liquid aliquots $(100 \mu \mathrm{L})$ were taken and diluted in $0.5 \mathrm{~g}$ of $2 \mathrm{wt} \% \mathrm{Cl}$-benzene in methanol as standard, and then analyzed by GC-FID and products were identified by GC-MS. Besides first-step condensation products as 
acetone, 3-pentanone and 2-methyl-2-pentenal, groups from 5 to $10 \mathrm{C}$ atoms molecules are distinguished and classified in $\mathrm{C} 5-\mathrm{C} 8$ and $\mathrm{C} 9-\mathrm{C} 10+$ to simplify their quantification. The method of group contributions was used to predict the corresponding FID response factor. Results of catalytic experiments have been expressed as total organic products yields as a percentage of maximum attainable (See SI).

$\S \S \S$ Recycling experiments: Once the catalytic experiment had finished, the solid was separated by centrifugation and washed repeatedly with methanol. After drying

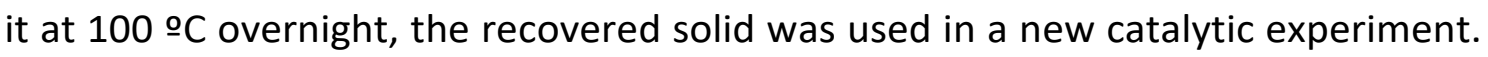
The solid was also analysed by EA and TG to measure the organic compounds deposition in the surface of the catalyst.

\section{References}

1 G. W. Huber, S. Iborra A. Corma, Chem. Rev., 2006, 106, 4044-4098.

2 C. O. Tuck, E. Pérez, I. T. Horváth, R. A. Sheldon, M. Poliakoff, Science, 2012, 337, 695699.

3 G. W. Huber, A. Corma, Angew. Chem., Int. Ed., 2007, 46(38), 7184-7201.

4 D. M. Alonso, J. Q. Bond, J. A. Dumesic, Green Chem., 2010, 12(9), 1493-1513.

5 A. Pinheiro, D. Hudebine, N. Dupassieux, C. Geantet, Energy Fuels, 2009, 23(2), 10071014

6 V. N. Bui, G. Toussaint, D. Laurenti, C. Mirodatos, C. Geantet, Catal. Today, 2009, 143(1-2), 172-178.

7 A. G. Gayubo, A. T. Aguayo, A. Atutxa, R. Prieto, J. Bilbao, Energy Fuels, 2004, 18, 16401647.

8 A. G. Gayubo, A. T. Aguayo, A. Atutxa, B. Valle, J. Bilbao, J. Chem. Technol. Biotechnol., $2005,80,1244-1251$.

9 D. C. Elliott, Energy Fuels, 2007, 21, 1792-1815. 
10 M. E. Domine, A. C. van Veen, Y. Schuurman, C. Mirodatos, ChemSusChem, 2008, 1, 179-181.

11 D. Radlein, A. Quignard, US Pat. 2014/0288338, 2014.

12 M. Asadieraghi, W. M. A. Wan Daud, H. F. Abbas, Renew. \& Sust. Ener. Rev., 2014, 36, 286-303.

13 R. D. Cortright, R. R. Davda, J. A. Dumesic, Nature, 2002, 418, 964-967.

14 J.W Shabaker, G.W Huber, J.A Dumesic, J. Catal., 2004, 222(1), 15180-15191.

15 F. Cherubini, G. Jungmeier, M. Wellisch, T. Willke, I. Skiadas, R. van Ree, E. de Jong, Biofuels, Bioprod. \& Bioref., 2009, 3(5), 534-546.

16 C. A. Gaertner, J.C. Serrano Ruiz, J. A. Dumesic, J. Catal., 2009, 266(1), 71-78.

17 T. Pham, T. Sooknoi, D.E. Resasco, J. Catal., 2012, 295, 169.

18 A. Gangadharan, M. Shen, T. Sooknoi, D. E. Resasco, R. G. Mallinson, Appl. Catal. A: Gen., 2010, 385(1-2), 80-91.

19 S. Wang, K. Goulas, E. Iglesia, J. Catal., 2016, 340, 302-320.

20 S. Wang, E. Iglesia, J. Catal., 2017, 345, 183-206.

21 N. Aranda-Pérez, M. P. Ruiz, J. Echave, J. Faria, Appl. Catal. A: Gral., 2017, 531, 106-118.

22 K. Nakajima, Y. Baba, R. Noma, M. Kitano, J. N. Kondo, S. Hayashi, M. Hara, J. Am.

Chem. Soc., 2011, 133(12), 4224-4227.

23 R. Rodriguez, D. Mandelli, N. S. Gonçalves, P. P. Pescarmona, W. Carvalho, J. Mol. Catal. A: Chem., 2016, 422, 122-130.

24 H. T. Kreissl, K. Nakagawa, Y-K. Peng, Y. Koito, J. Zheng, S. C. E. Tsang, J. Catal., 2016, 338, 329-339.

25 M. Paulis, M. Martín, D. B. Soria, A. Díaz, J. A. Odriozola, M. Montes, Appl. Catal. A: Gen., 1999, 180(1-2), 411-420.

26 G. S. Foo, D. Wei, D. S. Sholl, C. Sievers, ACS Catal., 2014, 4(9), 3180-3192.

27 M. E. Domine, J. M. López-Nieto, D. Delgado, A. Férnandez-Arroyo, ES Pat. P201630339, 2016.

28 J. C. Serrano-Ruiz, J. Luettich, A. Sepúlveda-Escribano, F. Rodríguez-Reinoso, J. Catal., 2006, 241(1), 45-55. 
29 K. Omata, K. Matsumoto, T. Murayama, W. Ueda, Catal. Today, 2016, 259(1), 205-212.

30 T. Murayama, J. Chen, J. Hirata, K. Matsumoto, W. Ueda, Catal. Sci. Technol., 2014, 4(12), 4250-4257. 
Table 1. Physicochemical and catalytic characteristics of representative catalysts.

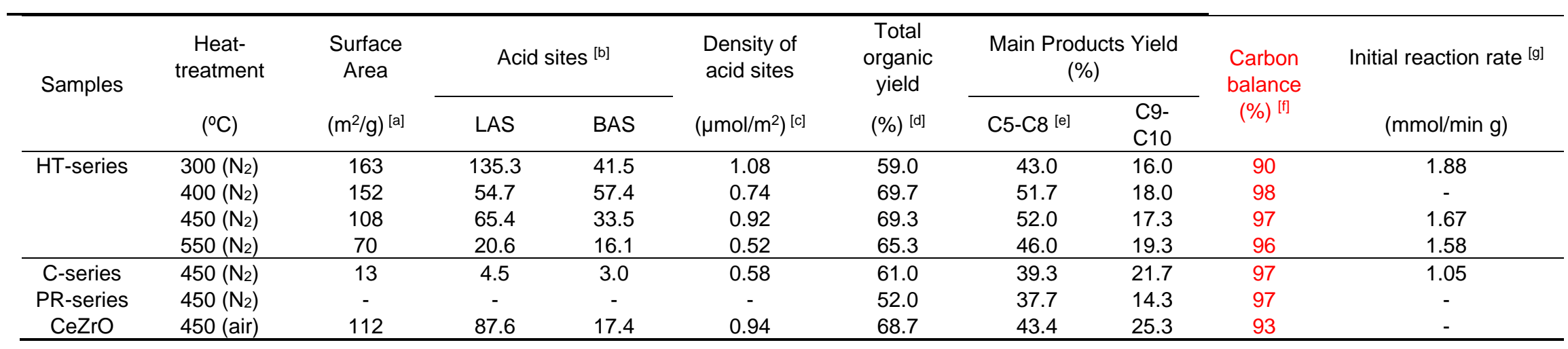

[a] Calculated values from $\mathrm{N}_{2}$ adsorption isotherms (BET method). [b] Values calculated by pyridine adsorption FT-IR measurements. [c] Density of total acid sites (BAS + LAS).

[d] Total organic product yield. [e] Sum of 2-methyl-2-pentenal and other C5-C8 products. [f] Carbon balance calculated from GC-FID. [g] Initial reaction rate values calculated at

$1 \mathrm{~h}$ of reaction (See SI). Reaction conditions: $3.0 \mathrm{~g}$ of aqueous model mixture, $0.15 \mathrm{~g}$ of catalyst at $200^{\circ} \mathrm{C}$ and $\mathrm{P}_{\mathrm{N} 2}=13 \mathrm{bar}$ during $7 \mathrm{~h}$. 


\section{Caption to Figures}

Figure 1. XRD patterns and TEM images of representative NbOx-based catalysts, heat-treated in $\mathrm{N}_{2}$ at $450 \circ \mathrm{C}$ : a) HT-450N; b) PR-450N; and c) C-450N.

Figure 2. a) Conversion of acetic acid, ethanol, propanal and acetol, and b) evolution of products yields vs reaction time over HT-450N catalyst. [Reaction conditions: $3.0 \mathrm{~g}$ of aqueous model mixture, $0.15 \mathrm{~g}$ of catalyst at $200 \stackrel{\circ}{ } \mathrm{C}$ and $\mathrm{P}_{\mathrm{N} 2}=$ 13 bar during $7 \mathrm{~h}]$.

Figure 3. Proposed reaction network. Initial mixture (orange), intermediate C5-C8 products (red) and final C9-C10 products (blue) are outlined.

Figure 4. Variation of total organic product yield, TOP (black) and intermediates, INT's (red) and C9-C10 products (blue) yields with the density of acid sites for all catalysts: HT-series (filled symbols); PR-series and C-series (empty symbols). Results over CeZrO sample (star symbols) are also included.

Figure 5. Effect of the re-uses (R0, R1 and R2) of different catalysts on the total organic yield (\%) attained during the condensation of oxygenated compounds in aqueous model mixture (At $200 \circ \mathrm{C}$ and $\mathrm{P}_{\mathrm{N} 2}=13$ bar during $7 \mathrm{~h}$ ). 